\title{
Psychiatric Correlates of Bullying in the United States: Findings from a National Sample
}

\author{
Michael G. Vaughn • Qiang Fu • Kimberly Bender • Matt DeLisi • \\ Kevin M. Beaver • Brian E. Perron • Matthew O. Howard
}

Published online: 23 February 2010

(C) Springer Science+Business Media, LLC 2010

\begin{abstract}
The aim of this study was to examine the psychiatric correlates of bullying behavior in the United States. Data were derived from the National Epidemiologic Survey on Alcohol and Related Conditions, a nationally representative sample of US adults. Structured psychiatric interviews $(N=43,093)$ were completed by trained lay interviewers between 2001 and 2002. Six percent of US adults reported a lifetime history of bullying others. Respondents who were men, 18 to 34 , Asian/Native American, earned $\leq \$ 35,000$ annually, were born in the US, and received no college education had significantly higher
\end{abstract}

M. G. Vaughn

School of Social Work, Saint Louis University, St. Louis, MO, USA

M. G. Vaughn

Division of Epidemiology, Department of Community Health, School of Public Health, Saint Louis University, St. Louis, MO, USA

M. G. Vaughn $(\bowtie)$

Tegeler Hall, 3550 Lindell Boulevard, St. Louis, MO 63103, USA

e-mail: mvaughn9@slu.edu

Q. Fu

Department of Biostatistics, School of Public Health, Saint Louis University, St. Louis, MO, USA

K. Bender

School of Social Work, University of Denver, Denver, CO, USA

M. DeLisi

Criminology and Criminal Justice Studies, Department of Sociology, Iowa State University, Ames, IA, USA

K. M. Beaver

College of Criminology and Criminal Justice, Florida State University, Tallahassee, FL, USA

B. E. Perron

School of Social Work, University of Michigan, Ann Arbor, MI, USA

M. O. Howard

School of Social Work, University of North Carolina, Chapel Hill, NC, USA 
rates of bullying. Multivariate logistic regression analyses identified significant associations between bullying and bipolar disorder, lifetime alcohol and marijuana use disorders, nicotine dependence, conduct disorder, antisocial, paranoid, and histrionic personality disorders, and family history of antisocial behavior. Prevention and treatment targeting bullying behaviors, comorbid conditions, and their precursors could potentially reduce the prevalence and consequences of bullying.

Keywords Aggression · Bullying · Comorbidity · Antisocial behavior · Violence

\section{Introduction}

Bullying, defined as aggressive behavior used to repeatedly harm or intimidate others with less power [1] often begins in childhood and affects approximately $30 \%$ of youth in the US [2]. Bullying involves an imbalance of power that manifests in acts of physical or relational aggression. While bullies may intimidate their victims through physical aggression such as kicking, punching or slapping, they may also use verbal threats, social exclusion, gossiping, and name-calling to assert their power over victims [2]. Longitudinal research clearly documents emotional consequences for victims of bulling, including low selfesteem [3], anxiety and passivity [4], academic problems [5], and social deficits [6]. With growing concern about the detrimental effects of bullying, recent research has focused on characterizing bullies themselves to further inform prevention efforts.

One national study found that approximately $19 \%$ of youth in the US reported bullying other people [2]. Bullying behavior during childhood is associated with mental health disorders, including depression, conduct disorder, oppositional defiant disorder, and attention deficit disorder [7, 8]. In addition, youth who bully others often exhibit passiveaggressive, histrionic, and paranoid personality traits [9]. Children who bully are also more likely to engage in subsequent delinquent behavior [10], leading some researchers to conclude that problem behavior is a consequence of bullying [11].

Studies examining characteristics of bullies among adult samples are limited. Although the majority of bullying takes place in childhood or adolescence [12], some individuals continue to bully others as adults [13]. Research indicates that bullying can be chronic, beginning in childhood and persisting into adulthood with detrimental effects on development and maintenance of healthy relationships [14]. Investigations of bullying utilizing adult samples can thus shed light on the consequences of childhood bullying and correlates of bullying in adulthood.

Whereas several studies have investigated bullying among adult prisoner populations [15-17] only a few longitudinal studies have examined adult correlates of bullying in the general population. A study in Finland followed elementary school-aged boys who bullied into adulthood [4]. This research suggested that being a bully can have long-term social and psychological consequences. Boys who bullied others evidenced significantly more prevalent antisocial personality disorder, criminality and higher conviction rates as adults than their non-bullying counterparts. Olweus [18] found that among middle school children who bullied, $70 \%$ were convicted of a crime by age 24 . In addition, bullying in childhood is a marker of enhanced risk for substance abuse, depression and anxiety in adulthood [4]. These findings suggest that psychiatric characteristics associated with bullying in youth persist into adulthood. However, studies of bullying among adults are limited in scope and 
relatively few such studies have been conducted in the US. A clear gap in this body of work is the absence of an epidemiological study of bullying among US adults.

\section{Study purpose}

Although prior studies suggest that persons who bully may have significant psychiatric problems as adults, limited empirical research has accrued on this relationship. Therefore, the purpose of this study was to examine the sociodemographic, psychiatric, and substance use correlates of bullying in a nationally representative sample of US adults. The primary aims were to (1) compare adults reporting a lifetime history of bullying to individuals without such a history with respect to sociodemographic variables, childhood and adult antisocial behaviors, and lifetime mood, anxiety, substance use, and personality disorders, and (2) to estimate the strength of the associations between these variables and bullying in controlled multivariate analyses.

\section{Methods}

Participants

Study findings are based on data from the 2001 to 2002 National Epidemiologic Survey on Alcohol and Related Conditions (NESARC). NESARC is a nationally representative sample of 43,093 non-institutionalized US residents aged 18 years and older [19]. The survey gathered background data and extensive information about substance use and comorbid psychiatric disorders, including personality disorders, from individuals living in households and group settings such as shelters, college dormitories, and group homes in all 50 states and the District of Columbia. NESARC utilized a multistage cluster sampling design, oversampling young adults, Hispanics, and African-Americans in the interest of obtaining reliable statistical estimation in these subpopulations, and to ensure appropriate representation of racial/ethnic subgroups. The overall response rate was $81 \%$. Data were weighted at the individual and household levels to adjust for oversampling and non-response on demographic variables (i.e., age, race/ethnicity, sex, region, and place of residence). Data were also adjusted to be representative (based on region, age, race, and ethnicity) of the US adult population as assessed during the 2000 Census. Study participants provided fully informed consent. The US Census Bureau and the US Office of Management and Budget approved the research protocol and informed consent procedures.

\section{Diagnostic Assessment}

Data were collected through face-to-face structured psychiatric interviews conducted by US Census workers trained by the National Institute on Alcohol Abuse and Alcoholism and US Census Bureau. Interviewers administered the Alcohol Use Disorder and Associated Disabilities Interview Schedule-DSM-IV version (AUDADIS-IV), which provides diagnoses for mood, anxiety, personality, and substance use disorders. The AUDADIS IV has shown to have good-to-excellent reliability in assessing alcohol and drug use in the general population $[20,21]$. 
Bullying

The lifetime prevalence of bullying was assessed with an item embedded in the conduct disorder and part of the antisocial behavior interview module. All NESARC participants were asked the following question: "In your ENTIRE life, did you EVER have a time when you bullied or pushed people around or tried to make them afraid of you?" NESARC respondents who answered yes were defined as having a history of bullying. There are few national measures of bullying and use of this single item measure is one of few available ways to look at prevalence nationally. Although the reliability of this individual item is unknown, the test-retest reliability for the antisocial personality disorder diagnosis was adequate $(r=0.69)$ [19]. The internal consistency reliability for the entire antisocial personality disorder criterion set was also good $(\alpha=0.86)$ [22].

Personality disorders

Consistent with current conceptualizations of personality disorders, including DSM-IV [23-25] personality disorder diagnoses reflected long-standing impairments, characteristic patterns of behavior, and exclusion of cases where substance use intoxication or withdrawal, other medication use, or physical illnesses could have contributed to reported Axis II personality disorder signs and symptoms. In addition to antisocial personality disorder, other personality disorders assessed included avoidant, dependent, obsessive-compulsive, paranoid, schizoid, and histrionic disorders.

\section{Psychiatric and Substance Use Disorders}

Numerous control variables were used to reduce confounding including lifetime alcohol (alcohol abuse/dependence) and drug (abuse/dependence on heroin, hallucinogens, cocaine/crack, marijuana, stimulants, painkillers, tranquilizers, and sedatives) use disorders, nicotine dependence, pathological gambling, and lifetime DSM-IV mood (major depression, dysthymia, and bipolar disorder) and anxiety (social phobia, generalized anxiety disorder, panic disorder, and specific phobia) disorders [26]. Family history of antisocial behavior based on any parental or sibling history of antisocial behavior was also assessed. We also included body mass index (BMI) categories (i.e., normal, overweight, obese) based on previous research on the relationship between BMI and bullying [27]. Response categories for region of residence in U.S., urbanicity, race/ethnicity, sex, age, marital status, educational background, unemployment status, and individual and family income are listed in Table 1.

\section{Statistical Analyses}

Weighted prevalence estimates and standard errors were computed using SUDAAN Version 9.0. [28] This system implements a Taylor series linearization to adjust standard errors of estimates for complex survey sampling design effects including clustered data. Multivariate logistic regression analyses were conducted to assess convergent relations of bullying to childhood and adult antisocial behaviors and to assess psychiatric disorders among persons who have reported bulling versus persons who have not reported bullying while controlling for sociodemographic covariates and lifetime psychiatric disorders. In this way, we were able to isolate the effect of each diagnostic category on our dependent variables. Adjusted odds ratios (AORs) and $95 \%$ confidence intervals are presented to 
Table 1 Sociodemographic characteristics of adult NESARC respondents with and without a lifetime history of bullying behavior

\begin{tabular}{|c|c|c|c|}
\hline Characteristic & $\begin{array}{l}\text { Bullying } \\
(N=2460) \\
\%(95 \% \mathrm{CI})\end{array}$ & $\begin{array}{l}\text { Non-bullying } \\
(N=39,501) \\
\%(95 \% \mathrm{CI})\end{array}$ & OR $(95 \% \mathrm{CI})$ \\
\hline \multicolumn{4}{|l|}{ Sex } \\
\hline Men & $65.18(62.97-67.33)$ & $46.67(46.04-47.30)$ & $2.07(1.28-1.88)$ \\
\hline Women & $34.82(32.67-37.03)$ & $53.33(52.70-53.96)$ & 1.00 \\
\hline \multicolumn{4}{|l|}{ Race } \\
\hline Hispanic & $10.84(8.73-13.38)$ & $11.58(9.29-14.34)$ & $0.94(0.77-1.15)$ \\
\hline \multicolumn{4}{|l|}{ Asian/Alaska/Indian } \\
\hline Native American & $6.82(5.47-8.49)$ & $6.45(5.49-7.58)$ & $1.41(1.10-1.81)$ \\
\hline African American & $11.54(9.79-13.56)$ & $10.97(9.75-12.31)$ & $0.92(0.79-1.06)$ \\
\hline White & $70.80(67.35-74.02)$ & $71.00(67.68-74.11)$ & 1.00 \\
\hline \multicolumn{4}{|l|}{ Nativity } \\
\hline Born in the US & 92.37 (90.13-94.13) & $84.96(81.65-87.77)$ & $2.81(2.20-3.61)$ \\
\hline Born in a foreign country & $7.63(5.87-9.87)$ & $15.04(12.23-18.35)$ & 1.00 \\
\hline \multicolumn{4}{|l|}{ Age (years) } \\
\hline $65+$ & $4.32(3.49-5.33)$ & $17.05(16.36-17.76)$ & $0.13(0.10-0.16)$ \\
\hline $50-64$ & $14.83(13.23-16.60)$ & $21.48(20.96-22.02)$ & $0.38(0.32-0.44)$ \\
\hline $35-49$ & $33.03(30.68-35.47)$ & $30.96(30.31-31.62)$ & $0.65(0.57-0.73)$ \\
\hline $18-34$ & $47.82(45.43-50.21)$ & $30.51(29.61-31.42)$ & 1.00 \\
\hline \multicolumn{4}{|l|}{ Education } \\
\hline Less than high school & $17.45(15.44-19.64)$ & $15.39(14.43-16.40)$ & $1.63(1.37-1.95)$ \\
\hline High school graduate & $33.44(31.12-35.84)$ & $29.03(27.92-30.16)$ & $1.35(1.21-1.51)$ \\
\hline Some college or higher & $49.11(46.52-51.72)$ & $55.59(54.30-56.86)$ & 1.00 \\
\hline \multicolumn{4}{|l|}{ Income } \\
\hline $0-19,999$ & $25.08(22.84-27.45)$ & 23.33 (22.39-24.30) & $1.38(1.17-1.63)$ \\
\hline $20,000-34,999$ & $21.05(19.27-22.94)$ & $20.06(19.40-20.73)$ & $1.20(1.04-1.40)$ \\
\hline $35,000-69,999$ & $32.87(30.45-35.39)$ & $32.15(31.49-32.81)$ & $1.10(0.94-1.29)$ \\
\hline $70,000+$ & $21.00(18.80-23.40)$ & $24.47(23.07-25.91)$ & 1.00 \\
\hline \multicolumn{4}{|l|}{ Marital status } \\
\hline Never married & $28.73(26.37-31.20)$ & $20.17(19.24-21.13)$ & $0.95(0.83-1.10)$ \\
\hline Widowed/separated/divorced & $14.45(12.67-16.43)$ & 17.55 (17.09-18.02) & $1.17(0.98-1.39)$ \\
\hline Married/cohabitating & $56.83(54.02-59.59)$ & $62.28(61.32-63.24)$ & 1.00 \\
\hline \multicolumn{4}{|l|}{ Urbanicity } \\
\hline Central city & $30.12(26.08-34.49)$ & $29.40(25.23-33.94)$ & $1.04(0.93-1.17)$ \\
\hline Rural/suburban & $69.88(65.51-73.92)$ & $70.60(66.06-74.77)$ & 1.00 \\
\hline \multicolumn{4}{|l|}{ Region } \\
\hline Northeast & $17.21(12.12-23.85)$ & $19.87(13.88-27.61)$ & $0.81(0.67-0.99)$ \\
\hline Midwest & $28.89(22.74-35.93)$ & $22.80(17.07-29.76)$ & $1.04(0.87-1.25)$ \\
\hline South & $30.22(24.72-36.36)$ & $35.45(29.19-42.25)$ & $0.71(0.59-0.86)$ \\
\hline West & $23.68(17.66-30.99)$ & $21.89(15.64-29.75)$ & 1.00 \\
\hline
\end{tabular}


Table 1 continued

\begin{tabular}{llll}
\hline Characteristic & $\begin{array}{l}\text { Bullying } \\
(N=2460) \\
\%(95 \% \mathrm{CI})\end{array}$ & $\begin{array}{l}\text { Non-bullying } \\
(N=39,501) \\
\%(95 \% \mathrm{CI})\end{array}$ & OR $(95 \% \mathrm{CI})$ \\
\hline BMI & & & \\
Obese & $32.07(29.73-34.52)$ & $42.42(41.51-43.34)$ & $\mathbf{0 . 5 8}(\mathbf{0 . 5 1 - 0 . 6 6 )}$ \\
Overweight & $37.69(35.50-39.92)$ & $34.49(33.85-35.12)$ & $\mathbf{0 . 8 1}(\mathbf{0 . 7 2 , \mathbf { 0 . 9 1 } )}$ \\
Normal & $30.24(27.91-32.68)$ & $23.09(22.31-23.90)$ & 1.00 \\
\hline
\end{tabular}

Note: $C I$ confidence interval, $O R$ odds ratio adjusted for sociodemographic variables. OR values in bold are statistically significant $(p<0.05)$

reflect association strength. Adjusted odds ratios were considered statistically significant only if associated confidence intervals did not include the value 1.0.

\section{Results}

Sociodemographic Characteristics of Bullying and Non-Bullying Respondents

Table 1 shows sociodemographic characteristics of adults with and without a lifetime history of bullying. Persons reporting a lifetime history of bullying were more likely to be men $(\mathrm{OR}=2.07,95 \% \mathrm{CI}=1.28-1.88)$, born in the $\mathrm{US}(\mathrm{OR}=2.81,95 \% \mathrm{CI}=2.20$ 3.61), and American Indian/Alaska Native or Asian/Native Hawaiian/Pacific Islander $(\mathrm{OR}=1.41,95 \% \mathrm{CI}=1.10-1.81)$. Compared to respondents $18-34$, persons 35 and older were less likely to report bullying behavior. With respect to educational attainment, persons with a high school education $(\mathrm{OR}=1.35,95 \% \mathrm{CI}=1.21-1.51)$ or less than a high school education $(\mathrm{OR}=1.63,95 \% \mathrm{CI}=1.37-1.95)$ were more likely to report bullying compared to individuals with some college education. Persons reporting annual income of $\leq \$ 35,000$ were more likely to report bullying behavior. Compared to persons from the western region of the US, individuals from the northeast $(\mathrm{OR}=0.81,95 \% \mathrm{CI}=0.67-$ $0.99)$ and south $(\mathrm{OR}=0.71,95 \% \mathrm{CI}=0.59-0.86)$ were significantly less likely to report a lifetime history of bullying. Persons who were overweight $(\mathrm{OR}=0.58,95 \% \mathrm{CI}=0.51-$ $0.66)$ and obese $(\mathrm{OR}=0.81,95 \% \mathrm{CI}=0.72-0.91)$ were significantly less likely to report bullying compared to the normal weight individuals. There were no significant differences with respect to urbanicity and marital status.

\section{Bullying and Associated Antisocial Behaviors}

The prevalence of all antisocial behaviors was substantially higher among persons with a lifetime history of bullying compared to persons without such a history (see Table 2). The prevalence of antisocial behaviors was typically three to five times greater for respondents reporting a lifetime history of bullying compared to respondents with no such history; thus, we controlled for the effect of other lifetime antisocial behaviors. These analyses showed significant associations for numerous behaviors including harassing and threatening (14.8, $95 \% \mathrm{CI}=12.97-16.8 \%, \mathrm{OR}=2.55,95 \% \mathrm{CI}=1.91-3.42)$, hitting someone so hard they were injured $(29.8,95 \% \mathrm{CI}=27.51-32.10 \%, \mathrm{OR}=1.80,95 \% \mathrm{CI}=1.49-2.18)$, cruelty to animals $(9.36,95 \% \mathrm{CI}=7.92-11.02 \%, \mathrm{OR}=1.95,95 \% \mathrm{CI}=1.44-2.64)$, and stealing 
Table 2 Convergent relations between antisocial behaviors in adults with and without a history of bullying behavior

\begin{tabular}{|c|c|c|c|}
\hline Behavior & $\begin{array}{l}\text { Bullying } \\
(N=2,458) \\
\%(95 \% \text { CI })\end{array}$ & $\begin{array}{l}\text { Non-bullying } \\
(N=39,422) \\
\%\left(95 \% \mathrm{CI}^{\mathrm{a}}\right)\end{array}$ & OR $95 \% \mathrm{CI}$ \\
\hline
\end{tabular}

Cut class and leave without permission ${ }^{\mathrm{a}}$

Stay out late at night ${ }^{\mathrm{a}}$

Set a fire on purpose ${ }^{\mathrm{a}}$

Run away from home overnight ${ }^{\mathrm{a}}$

Be absent from work/school a lot ${ }^{\mathrm{a}}$

Quit a job without knowing where to find another

Quit a school program without knowing what to do next ${ }^{\mathrm{a}}$

Travel around more than 1 month without plans

Have no regular place to live at least 1 month

Live with others at least 1 month

Lie a $\operatorname{lot}^{\mathrm{a}}$

Use a false or made up name/alias

Scam/con someone for money

Do things that could have easily hurt you/ others

Get three or more traffic tickets for reckless driving/causing accidents

Have a driver's license suspended/revoked

Hurt an animal on purpose $\mathrm{a}^{\mathrm{a}}$

Destroy others' property ${ }^{\mathrm{a}}$

Fail to pay off your debts

Steal anything from others

Forge someone's signature

Shoplift

Rob/mug someone or snatch a purse

Make money illegally

Do something you could have been arrested for

Force someone to have sex

Get into lots of fights that you started

Get into a fight that came to swapping blows with Husband/wife or boyfriend/girlfriend

Use a weapon in a fight

Hit someone so hard that you injure them

Harass/threaten/blackmail someone
$57.66(54.86-60.41) \quad 19.56(18.88-20.26) \quad 1.88$ (1.61-2.19)

61.76 (59.17-64.28) 23.38 (22.54-24.25) $\quad \mathbf{2 . 0 9}$ (1.82-2.40)

$\begin{array}{ccc}6.17(5.05-7.50) & 0.79(0.67-0.94) & 0.96(0.63-1.46) \\ 18.99(17.15-20.97) & 4.22(3.93-4.52) & 1.03(0.83-1.27) \\ 26.17(23.85-28.63) & 5.61(5.28-5.97) & 1.17(0.96-1.43) \\ 33.49(31.20-35.85) & 10.16(9.62-10.72) & \mathbf{1 . 3 0}(\mathbf{1 . 1 1 - 1 . 5 3 )}\end{array}$

$13.78(12.07-15.70) \quad 3.15(2.86-3.46) \quad 1.03(0.80-1.33)$

$11.96(10.27-13.88) \quad 2.88(2.65-3.12) \quad 0.74(0.54-1.01)$

$10.42(8.73-12.40) \quad 2.26(2.04-2.51) \quad \mathbf{0 . 6 7}(\mathbf{0 . 4 9 - 0 . 9 3 )}$

$30.70(28.29-33.22) \quad 9.88(9.24-10.55) \quad \mathbf{1 . 3 9}(\mathbf{1 . 1 8}-\mathbf{1 . 6 4})$

$23.44(21.38-25.63) \quad 4.08(3.82-4.35) \quad 9.55$ (7.17-12.71)

$10.22(8.76-11.90) \quad 1.62(1.46-1.79) \quad 1.00(0.72-1.39)$

$11.18(9.61-12.98) \quad 0.87(0.75-1.01) \quad 1.47(\mathbf{1 . 0 5}-\mathbf{2 . 0 7})$

40.85 (38.23-43.52) 12.35 (11.48-13.28) 1.33 (1.15-1.53)

$23.01(21.02-25.12) \quad 7.74(7.15-8.36) \quad 1.19(0.99-1.43)$

$22.49(20.42-24.71) \quad 6.80(6.33-7.30) \quad \mathbf{1 . 2 2}(\mathbf{1 . 0 0 - 1 . 4 8 )}$

$9.36(7.92-11.02) \quad 1.33(1.18-1.50) \quad \mathbf{1 . 9 5}(\mathbf{1 . 4 4 - 2 . 6 4 )}$

$20.77(18.67-23.05) \quad 2.61(2.36-2.88) \quad 1.27(0.97-1.66)$

$15.71(13.94-17.66) \quad 3.41(3.13-3.72) \quad 1.13(0.91-1.41)$

$35.02(32.55-37.57) \quad 7.27(6.78-7.79) \quad 1.59(\mathbf{1 . 3 1 - 1 . 9 2})$

$9.11(7.83-10.59) \quad 1.69(1.52-1.88) \quad 1.00(0.74-1.37)$

$37.02(34.61-39.49) \quad 9.63(9.00-10.31) \quad 1.14(0.95-1.37)$

$2.36(1.75-3.16) \quad 0.16(0.12-0.22) \quad 0.78(0.37-1.64)$

$14.14(12.41-16.07) \quad 2.01(1.82-2.23) \quad 0.63(0.47-0.84)$

48.58 (45.78-13.33) 13.33 (12.55-14.16) $1.30(\mathbf{1 . 1 0 - 1 . 5 2 )}$

$\begin{array}{ccc}0.74(0.40-1.36) & 0.10(0.02-0.14) & 1.43(0.53-3.86) \\ 25.38(23.34-27.53) & 1.31(1.18-1.46) & \mathbf{6 . 2 3}(\mathbf{5 . 1 5}-\mathbf{7 . 5 4}) \\ 22.46(20.49-24.56) & 5.58(5.21-5.98) & \mathbf{1 . 3 0}(\mathbf{1 . 0 9 - 1 . 5 5}) \\ 15.39(13.60-17.38) & 1.83(1.67-2.01) & 1.18(0.89-1.57) \\ 29.76(27.51-32.10) & 4.44(4.12-4.79) & \mathbf{1 . 8 0}(\mathbf{1 . 4 9 - 2 . 1 8}) \\ 14.77(12.97-16.76) & 0.86(0.75-0.99) & \mathbf{2 . 5 5}(\mathbf{1 . 9 1 - 3 . 4 2})\end{array}$

Note: $C I$ confidence interval. OR odds ratio adjusted for all antisocial behaviors. OR values in bold are

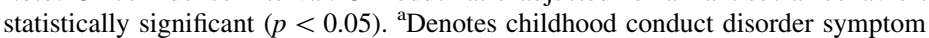


(35.0, 95\% CI $=32.55-37.57 \%, \mathrm{OR}=1.59,95 \% \mathrm{CI}=1.31-1.92)$. Large effects were found for lying $(\mathrm{OR}=9.55,95 \% \mathrm{CI}=7.17-12.71)$ and getting into fights that you started $(\mathrm{OR}=6.23,95 \% \mathrm{CI}=5.15-7.5)$. The least prevalent behavior was forcing someone to have sex $(0.74,95 \% \mathrm{CI}=0.40-1.36 \%)$.

Multivariate Logistic Regression Analysis Examining Associations between Bullying and Lifetime Psychiatric Comorbidity

Table 3 compares prevalence rates of lifetime psychiatric comorbidity and for persons reporting and not reporting a history of bullying. Odd ratios are adjusted for sociodemographic factors (i.e., race, sex, education, marital status, age, income, region, and urbanicity), previously described lifetime DSM-IV psychiatric disorders, and family history of antisocial behavior. The most prevalent psychiatric disorders among persons with a history of bullying were any lifetime alcohol use disorder $(62.58 \%, \mathrm{CI}=59.64-65.43 \%)$, nicotine dependence $(41.04 \%, \mathrm{CI}=38.33-43.82 \%)$, major depressive disorder $(30.87 \%$, $\mathrm{CI}=28.45-33.39 \%)$, antisocial personality disorder (29.49\%, CI $=26.98-32.13 \%)$, and marijuana use disorder $(28.43 \%, \mathrm{CI}=25.93-31.07 \%)$. A family history of antisocial behavior was also highly prevalent among persons reporting a history of bullying $(47.31 \%$, $\mathrm{CI}=44.57-50.06 \%$ ). Following adjustments, significant associations were found for lifetime bipolar disorder $(\mathrm{OR}=1.47,95 \% \mathrm{CI}=1.20-1.80)$, alcohol use disorder $(\mathrm{OR}=1.87,95 \% \mathrm{CI}=1.62-2.17)$, nicotine dependence $(\mathrm{OR}=1.40,95 \% \mathrm{CI}=1.21-$ 1.61), marijuana use disorder $(\mathrm{OR}=1.33,95 \% \mathrm{CI}=1.10-1.60)$, conduct disorder $(\mathrm{OR}=10.75,95 \% \mathrm{CI}=8.23-14.03)$, paranoid $(\mathrm{OR}=1.81,95 \% \mathrm{CI}=1.40-2.34)$, antisocial $(\mathrm{OR}=7.74, \quad 95 \% \quad \mathrm{CI}=6.47-9.27), \quad$ and histrionic $\quad(\mathrm{OR}=1.50, \quad 95 \%$ $\mathrm{CI}=1.07-2.11)$ personality disorders, and family history of antisocial behavior $(\mathrm{OR}=1.61,95 \% \mathrm{CI}=1.40-1.85)$.

\section{Discussion}

To our knowledge, this is the largest national epidemiological study examining the association between bullying and psychiatric diagnoses. The overall reported prevalence of bullying was six percent. Findings indicated that the prevalence of bullying varied by sociodemographic status, was associated with a broad array of antisocial behaviors, and, following adjustments for numerous confounding variables, was associated with several lifetime psychiatric diagnoses across multiple DSM diagnostic categories. Specifically, the current investigation found that young men living in the Western region of the US who had comparatively low levels of education and low annual incomes were at highest risk for bullying. That said, one in every 17 adults in the US reported a lifetime history of bullying other persons, indicating a high base rate for this form of antisocial behavior. Two additional empirical trends were particularly important. First, persons born in the US were nearly three times more likely than persons born outside of the US to report bullying behavior. This finding suggests that there is something about American culture that may promote bullying behavior (e.g., a widely accepted individualist or competitive orientation) or there could be greater stigma among persons from particular countries outside the US to admit their bullying behavior. Second, persons who are overweight and obese show decreased bullying. This finding converges with recent research that suggests that via social acceptance mechanisms obese individuals are often victims [27], indicating that weight segments the population, separating the powerful (normal BMI) from the 
Table 3 Psychiatric comorbidities of adults with and without a lifetime history of bullying behavior

\begin{tabular}{|c|c|c|c|}
\hline Comorbid psychiatric disorder & $\begin{array}{l}\text { Bullying } \\
(N=2460) \\
\%(95 \% \mathrm{CI})\end{array}$ & $\begin{array}{l}\text { Non-bullying } \\
(N=39,501) \\
\%(95 \% \mathrm{CI})\end{array}$ & $\begin{array}{l}\text { Adjusted odds ratios } \\
\text { OR }(95 \% \mathrm{CI})\end{array}$ \\
\hline \multicolumn{4}{|l|}{ Mood disorders } \\
\hline Major depressive disorder & $30.87(28.45-33.39)$ & $15.92(15.25-16.62)$ & $1.07(0.91-1.26)$ \\
\hline Bipolar disorder & $20.12(18.17-22.23)$ & $4.81(4.51-5.13)$ & $1.47(1.20-1.80)$ \\
\hline Dysthymia & $10.00(8.60-11.61)$ & $3.98(3.72-4.27)$ & $0.97(0.73-1.28)$ \\
\hline \multicolumn{4}{|l|}{ Anxiety disorders } \\
\hline Panic disorder & $8.16(6.92-9.61)$ & $3.81(3.55-4.10)$ & $1.06(0.81-1.37)$ \\
\hline Social phobia & $10.21(8.79-11.84)$ & $4.75(4.38-5.15)$ & $0.79(0.61-1.03)$ \\
\hline Specific phobia & $16.84(14.78-19.12)$ & $9.13(8.56-9.73)$ & $1.05(0.85-1.29)$ \\
\hline Generalized anxiety disorder & $9.40(8.00-11.01)$ & $3.89(3.58-4.22)$ & $1.07(0.84-1.37)$ \\
\hline \multicolumn{4}{|l|}{ Substance use disorders } \\
\hline Alcohol use disorder & $62.58(59.64-65.43)$ & $28.23(26.78-29.72)$ & $1.87(1.62-2.17)$ \\
\hline Nicotine dependence & $41.04(38.33-43.82)$ & $16.37(15.48-17.31)$ & $1.40(1.21-1.61)$ \\
\hline Marijuana use disorder & $28.43(25.93-31.07)$ & $7.19(6.73-7.68)$ & $1.33(1.10-1.60)$ \\
\hline Other illicit drug use disorder & $19.36(17.22-21.71)$ & $4.37(4.04-4.72)$ & $1.03(0.82-1.29)$ \\
\hline Psychotic disorder & $2.68(1.88-3.81)$ & $0.67(0.59-0.78)$ & $1.21(0.75-1.95)$ \\
\hline Conduct disorder & $6.99(5.84-8.35)$ & $0.68(0.58-0.81)$ & $10.75(8.23-14.03)$ \\
\hline \multicolumn{4}{|l|}{ Personality disorders } \\
\hline Avoidant & $7.30(5.87-9.03)$ & $2.08(1.89-2.29)$ & $1.12(0.80-1.58)$ \\
\hline Dependent & $1.83(1.18-2.84)$ & $0.41(0.34-0.51)$ & $0.54(0.25-1.17)$ \\
\hline Obsessive-compulsive & $19.32(17.49-21.30)$ & $7.32(6.89-7.76)$ & $1.17(0.97-1.41)$ \\
\hline Paranoid & $16.79(14.92-18.83)$ & $3.70(3.44-3.97)$ & $1.81(1.40-2.34)$ \\
\hline Schizoid & $9.16(7.70-10.87)$ & $2.80(2.59-3.04)$ & $0.96(0.71-1.29)$ \\
\hline Antisocial & $29.49(26.98-32.13)$ & $2.00(1.81-2.20)$ & $7.74(6.47-9.27)$ \\
\hline Histrionic & $8.53(7.12-10.20)$ & $1.44(1.30-1.58)$ & $1.50(1.07-2.11)$ \\
\hline Pathological gambling & $1.98(1.31-2.99)$ & $0.33(0.27-0.40)$ & $1.83(0.96-3.47)$ \\
\hline Family history of antisocial behavior & $47.31(44.57-50.06)$ & $21.41(20.41-22.46)$ & $1.61(1.40-1.85)$ \\
\hline \multicolumn{4}{|l|}{ BMI } \\
\hline Obese & $32.07(29.73-34.52)$ & $42.42(41.51-43.34)$ & $0.58(0.50-0.72)$ \\
\hline Overweight & $37.69(35.50-39.92)$ & $34.49(33.85-35.12)$ & $0.84(0.72,0.97)$ \\
\hline Normal & $30.24(27.91-32.68)$ & $23.09(22.31-23.90)$ & 1.00 \\
\hline
\end{tabular}

Note: $C I$ confidence interval, $O R$ odds ratio adjusted for sociodemographic variables (race, sex, education, marital status, age, income, region, ubanicity), lifetime psychiatric disorders (alcohol abuse/dependence, drug abuse/dependence on heroin, hallucinogens, cocaine/crack, marijuana, stimulants, painkillers, tranquilizers, and sedatives, nicotine dependence, major depression, dysthymia, bipolar disorder, social anxiety disorder, generalized anxiety disorder, panic disorder, specific phobia), and a family history of antisocial behavior. OR values in bold are statistically significant $(p<0.05)$

disempowered (high BMI or obese). However, the fact that obese people are more likely to be victims may not explain the fact that they are less likely to bully, or at least totally explain this finding. It could be that obese persons are less well equipped to bully, given that they are in less good physical condition or that they have lower testosterone as a result of being obese. 
Findings with Respect to Antisocial Behaviors

An additional finding is that bullying is associated with a broad array of antisocial behaviors such as getting into numerous physical altercations, school attendance problems, lying, cruelty to animals, stealing, and harassment. As such, bullying can be viewed as a marker for potential antisocial behavior syndromes including conduct disorder and Antisocial Personality Disorder. Multivariate analyses, controlling for sociodemographic, lifetime psychiatric disorders, and family history of antisocial behavior, demonstrated that individuals with a lifetime history of bullying were nearly eleven times more likely to possess a conduct disorder diagnosis and nearly eight times more likely to meet criteria for antisocial personality disorder than their non-bullying peers. These findings support general theoretical viewpoints which suggest that bullying is a marker for participation in a wide variety of violent and non-violent behaviors.

Findings with Respect to Substance Use and Personality Disorders

There was significant comorbidity between bullying and alcohol use disorder, cannabis use disorder, and nicotine dependence. These effects were relatively modest and ranged from odds ratios of 1.33 to 1.87 . There was also significant comorbidity between bullying and bipolar disorder $(\mathrm{OR}=1.47, \mathrm{CI}=1.20-1.80)$, paranoid $(\mathrm{OR}=1.81, \mathrm{CI}=1.40-2.34)$, and histrionic $(\mathrm{OR}=1.50, \mathrm{CI}=1.07-2.11)$ personality disorders. Bipolar disorder can be associated with significant affective lability including rage responses that include interpersonal aggression. Likewise, prior research suggests that paranoid youth and adults may misperceive innocuous behaviors on the part of others as provocative and respond inappropriately with interpersonally aggressive behaviors. Histrionic personality disorder is also characterized by inappropriately provocative behavior that could serve as a stimulus for aggressive counter responses. Other mood and anxiety disorders were not associated with lifetime bullying. This suggests that persons who bully are severely antisocial with important psychiatric impairments as suggested by prior research [4].

\section{Treatment and Prevention Implications}

In recognizing the serious consequences of bullying, several anti-bullying models have been developed to reduce or prevent bullying behavior during elementary and middle school. A recent systematic review across school-based anti-bullying interventions found programs to effectively reduce bullying between 17 and 23\% and highlighted the Olweus model as particularly effective in reducing bullying [29]. The Olweus model targets multiple systems in an effort to reduce bullying, including establishing school-wide antibullying policies, training teachers to address precipitating incidents in the classroom, and offering informational workshops to parents [30]. Findings from the present study suggest that effective prevention efforts are needed to reduce the antisocial behaviors and psychiatric impairments associated with bullying.

\section{Limitations}

Current study findings require interpretation within the context of several limitations. First, given that the study data are cross-sectional, temporal ordering or variables does not permit firm conclusions regarding causal determinants. As such, reported findings cannot clarify the etiologic relationship between bullying and its correlates. For example, the use and 
abuse of alcohol may be associated with bullying behavior due to its disinhibiting effects on neuroregulatory processes, thus facilitating an increase in bullying behaviors. Conversely, the propensity to bully may also involve particular phenotypic characteristics that also include the propensity toward antisocial behavior in general, including alcohol abuse. However, findings do suggest that bullying and psychopathology are intertwined. Longitudinal study designs beginning earlier in the life course that examine gene-environment interactions dynamically over time provide one way to elucidate the causal structure of bullying behavior. An additional limitation is that the NESARC excludes persons under age 18 and therefore relies on retrospective respondent recall of bullying over potentially long periods of time. This could lead to underreporting or to biased reporting with younger respondents recalling better than older respondents. Although the NESARC is a nationally representative sample, it is uncertain how the association between bullying and psychiatric and substance use disorders would be similar or different if enriched correctional or clinical samples were employed. In addition, the data on bullying did not include important contextual, situational, and precipitating information which is important to understanding the nature of bullying behaviors. Future studies on bullying would benefit from including these natural history features in such assessments. Despite these limitations, findings from this study provide new and important epidemiologic insights into the problem of bullying in the United States.

Acknowledgment NESARC was funded by the National Institute on Alcohol Abuse and Alcoholism with additional support provide by the National Institute on Drug Abuse. The authors are greatful for support from NIH grants: DA021405 (Dr. Howord) and K0CA104119 (Dr. Fu). The authors report no conflicts of interest.

\section{References}

1. Olweus D, Limber S, Mihalic S: Blueprints for Violence Prevention, Book Nine: Bullying Prevention Program. Boulder, CO, Center for the Study and Prevention of Violence, 1999

2. Nansel TR, Overpeck M, Pilla RS, et al.: Bullying behaviors among US youth: Prevalence and association with psychosocial adjustment. Journal of American Medical Association 285:2094-2100, 2001

3. Bond L, Carlin J, Thomas L, et al.: Does bullying cause emotional problems? A prospective study of young teenagers. British Medical Journal 323:480-484, 2001

4. Sourander A, Jenson P, Ronning JA, et al.: What is the early adulthood outcome of boys who bully or are bullied in childhood? The Finnish "from boy to a man" study. Pediatric 120:397-404, 2007

5. Glew GM, Fan M, Katon W, et al.: Bullying and school safety. The Journal of Pediatrics 152:123-128, 2008

6. Eslea M, Menesini E, Morita Y, et al.: Friendship and loneliness among bullies and victims: Data from seven countries. Aggressive Behavior 30:71-83, 2003

7. Kumpulainen K, Rasanen E, Puura K: Psychiatric disorders and the use of mental health services among children involved in bullying. Aggressive Behavior 27:102-110, 2001

8. Kumpulainen K, Rasanen E, Henttonen I: Children involved in bullying: Psychological disturbance and persistence of involvement. Child Abuse and Neglect 23:1253-1261, 1999

9. Coolidge FL, DenBoer JW, Segal DL: Personality and neuropsychological correlates of bullying behavior. Personality and Individual Differences 36:1559-1569, 2004

10. White NA, Loeber R: Bullying and special education as predictors of serious delinquency. Journal of Research in Crime and Delinquency 45:380-397, 2008

11. Kim YS, Leventhal BL, Koh YJ, et al.: School bullying and youth violence: Causes or consequences of psychopathology? Archives of General Psychiatry 63:1035-1041, 2006

12. Carney AG, Merrell KW: Bullying in schools: Perspective on understanding and preventing an international problem. School Psychology International 22:364-382, 2001

13. Einarssen S, Skogstad A: Epidemiological findings of bullying. European Journal of Work and Organizational Psychology 5:185-201, 1996 
14. Oliver R, Hoover JH, Hazler R: The perceived roles of bullying in small-town Midwestern schools. Journal of Counseling and Development 72:416-419, 1994

15. Ireland JL: Bullying behaviors among male and female prisoners: A study of adult and young offenders. Aggressive Behavior 25:161-178, 1999

16. Ireland JL: Distinguishing the perpetrators and victims of bullying behavior in a prison environment: A study of male and female adult prisoners. Legal and Criminological Psychology 6:229-246, 2001

17. Ireland JL, Power CL: Attachment, emotional loneliness, and bullying behavior: A study of adult and young offenders. Aggressive Behavior 30:298-312, 2004

18. Olweus D: Bully/victim problems in school: Facts and intervention. European Journal of Psychology of Education 12:495-510, 1997

19. Grant B F, Dawson DA, Stinson FS, et al.: The alcohol use disorder and associated disabilities interview schedule-IV (AUDADIS-IV): Reliability of alcohol consumption, tobacco use, family history of depression and psychiatric diagnostic modules in a general population sample. Drug and Alcohol Dependence 71:7-16, 2003

20. Grant BF, Harford T, Dawson DA, et al.: The alcohol use disorder and associated disabilities interview schedule (AUDADIS): Reliability of alcohol and drug modules in a general population sample. Drug and Alcohol Dependence 39:37-44, 1995

21. Hasin D, Carpenter KM, McCloud S, et al.: The alcohol use disorders and associated disabilities interview schedule (AUDADIS): Reliability of alcohol and drug modules in a clinical sample. Drug and Alcohol Dependence, 44:133-141, 1997

22. Blanco C, Grant J, Petry NM, et al.: Prevalence and correlates of shoplifting in the United States: Results from the national epidemiologic survey on alcohol and related conditions (NESARC). American Journal of Psychiatry 165:905-913, 2008

23. Goldstein RB, Grant BF, Juan WJ, et al.: Antisocial personality disorder with childhood- vs. adolescence-onset conduct disorder: Results from the national epidemiologic survey on alcohol and related conditions. Journal of Nervous and Mental Disorders 194:667-675, 2006

24. Grant BF, Hasin D, Stinson FS, et al.: Prevalence, correlates, and disability of personality disorders in the United States: Results from the national epidemiologic survey of alcohol and related conditions. Journal of Clinical Psychiatry 65:948-958, 2004

25. Grant BF, Stinson FS, Hasin DS, et al.: Co-occurrence of 12-month alcohol and drug use disorders and personality disorders in the United States: Results from the national epidemiologic survey on alcohol and related conditions. Archives of General Psychiatry 61:361-368, 2004

26. American Psychaitric Association: Diagnostic and Statistical Manual of Mental Disorders, Fourth edn., Text Revision. Washington, DC, Author, 2000

27. Janssen I, Craig WM, Boyce WF, et al.: Associations between overweight and obesity with bullying behavior in school-aged children. Pediatrics 113:1187-1194, 2004

28. Research Triangle Institute: Software for survey data analysis, SUDAAN. Version 9.0. Research Triangle Park, NC, Research Triangle Institute, 2004

29. Ttofi MM, Farrington DP, Baldry AC: Effectiveness of Programmes to Reduce School Bullying: A Systematic Review. Stockholm, Sweden, The National Council for Crime Prevention, 2008

30. Olweus D: The Olweus Bullying Prevention Programme: Design and Implementation Issues and A New National Initiative in Norway. In Smith PK, Pepler D, Rigby K (Eds) Bullying in Schools: How Successful can Interventions be? Cambridge, Cambridge University Press, pp. 13-36, 2004

\section{Author Biographies}

Michael G. Vaughn, Ph.D. is currently Assistant Professor in the School of Social Work and Holds appointments in Public Policy and Epidemiology at Saint Louis University. His research includes antisocial behavior over the life-course with particular attention to psychopathy, substance abuse, self-regulation, and violence.

Qiang Fu, M.D., Ph.D. is an Associate Professor in Community Health and the Director of Biostatistics Division at Saint Louis University School of Public Health. His research interests include latent variable analysis, longitudinal data analysis, behavior genetics, and psychiatric epidemiology of externalizing behaviors and substance use disorders.

Kimberly Bender, Ph.D. is Assistant professor in the Graduate School of Social Work at the University of Denver. Her interests involve trauma and victimization during childhood and adolescence. 
Matt DeLisi, Ph.D. is Coordinator of Criminal Justice Studies at Iowa State University.

Kevin M. Beaver, Ph.D. is an Assistant Professor in the College of Criminology and Criminal Justice at Florida State University. He is the recipient of the American Society of Criminology's Ruth Shonle Cavan Young Scholar Award and the National Institute of Justice's Graduate Research Fellowship.

Brian E. Perron, Ph.D. is an Assistant Professor at the University of Michigan, School of Social Work. His research focuses on issues related to the serious mental illnesses and substance use disorders. He is involved in a variety of research activities, including analysis of nationally representative data and clinic-based surveys, and collaborating on field-based interventions.

Matthew O. Howard, Ph.D. is currently Frank A. Daniels, Jr. Distinguished Professor of Human Services Policy at the School of Social Work at the University of North Carolina at Chapel Hill. Dr. Howard has published 135 articles, received three grants from the National Institute on Drug Abuse. His research interests involve adolescent substance abuse and delinquency. 